\title{
Individual and joint effects of trehalose and glutamate on diabetic retinopathy: a propensity score-matched case-control study
}

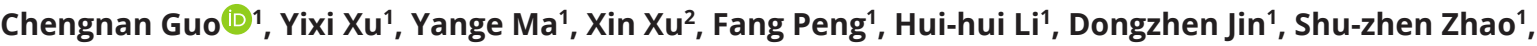 \\ Zhezheng Xia ${ }^{1}$, Mengyuan Lai ${ }^{1}$, Mingzhu Che ${ }^{1}$, Ruogu Huang ${ }^{1}$, Yanan Wang ${ }^{1}$, Depeng Jiang ${ }^{3}$, Chao Zheng ${ }^{4}$ and \\ Guangyun Mao',5,6
}

\author{
${ }^{1}$ Division of Epidemiology and \\ Wenzhou, Zhejiang, China \\ ${ }^{2}$ Wenzhou Medical University, Wenzhou, Zhejiang, China \\ ${ }^{3}$ Department of Community Health Sciences, College of Medicine, University of Manitoba, Winnipeg, Canada \\ ${ }^{4}$ The Second Affiliated Hospital of Zhejiang University School of Medicine, Hangzhou, Zhejiang, China \\ ${ }^{5}$ Eye Hospital and School of Ophthalmology and Optometry, Wenzhou Medical University, Wenzhou, Zhejiang, China \\ ${ }^{6}$ National Clinical Research Center for Ocular Diseases, Wenzhou, Zhejiang, China
}

Correspondence should be addressed to C Zheng or G Mao: chao_zheng@zju.edu.cn or mgy@wmu.edu.cn

\begin{abstract}
Although previous studies demonstrate that trehalose can help maintain glucose homeostasis in healthy humans, its role and joint effect with glutamate on diabetic retinopathy (DR) remain unclear. We aimed to comprehensively quantify the associations of trehalose and glutamate with DR. This study included 69 pairs of DR and matched type 2 diabetic (T2D) patients. Serum trehalose and glutamate were determined via ultraperformance liquid chromatography-electrospray ionization-tandem mass spectrometry system. Covariates were collected by a standardized questionnaire, clinical examinations and laboratory assessments. Individual and joint association of trehalose and glutamate with DR were quantified by multiple conditional logistic regression models. The adjusted odds of DR averagely decreased by $86 \%$ (odds ratio (OR): $0.14 ; 95 \% \mathrm{Cl}: 0.06,0.33$ ) with per interquartile range increase of trehalose. Comparing with the lowest quartile, adjusted OR $(95 \% \mathrm{Cl})$ were $0.20(0.05,0.83), 0.14(0.03,0.63)$ and $0.01(<0.01,0.05)$ for participants in the second, third and fourth quartiles of trehalose, respectively. In addition, as compared to their counterparts, T2D patients with lower trehalose (<median) and higher glutamate ( $\geq$ median) had the highest odds of DR (OR: 36.81; 95\% Cl: 6.75, 200.61). An apparent supermultiplicative effect of trehalose and glutamate on DR was observed, whereas relative excess risk due to interaction was not significant. The study suggests that trehalose is beneficial to inhibit the occurrence of DR and synergistically decreases the risk of DR with reduced glutamate. Our findings also provide new insights into the mechanisms of DR and further longitudinal studies are required to confirm these findings.
\end{abstract}

\author{
Key Words \\ - diabetic retinopathy \\ - glutamate \\ - trehalose \\ - joint effect \\ - propensity score matching
}

\section{Introduction}

As the primary cause of preventable vision loss in workingaged people, diabetic retinopathy (DR) has become one of the major public health issues worldwide $(1,2)$. From 1990 to 2015, the crude prevalence of DR-caused visual impairment and blindness has been gradually increased (3) and induced significant health threats to people and financial burden to the society (4), especially in some low- and middle-income countries. The overall age-

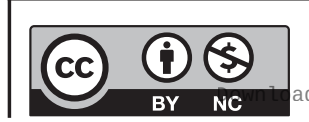

This work is licensed under a Creative Commons Attribution-NonCommercial 4.0 International License. ded from Bioscientifica.com at 04/26/2023 02:19:44PM
Endocrine Connections (2022) 11, e210474 
standardized DR prevalence in diabetic patients from 1980 to 2008 has been evaluated as $34.6 \%$ (95\% CI, 34.5, 34.8) according to a global meta-analysis (5). While the annual incidence of DR between 1980 and 2018 is assessed within $2.2-12.7 \%$ in accordance with another systematic review (6). Previous studies report that the prevalence of DR mainly increases with three clinical characteristics, including prolonged duration of diabetes, constant hyperglycemia (high glycated hemoglobin (HbA1c), and hypertension (10 mmHg decreased systolic blood pressure (SBP) roughly decreases $35 \%$ risk of DR progression) $(1,3,5)$. Increasingly, evidence suggests that retinal neurodegeneration plays a key role in the early pathogenesis of DR though the classic characteristic of DR is microcirculatory impairment $(7,8,9,10)$.

Trehalose is a naturally neuroprotective disaccharide, cannot synthesize on its own in the human body and is widely used as a food stabilizer, especially in many Asian countries $(11,12)$. Ingested trehalose is mainly degraded and absorbed into the body by trehalase (13), a hydrolytic enzyme regulated by the trehalase gene (TREH) (14). It has been proved that trehalose is beneficial to neuroprotection and antiaging (11), and is effective in preventing metabolic syndrome (15), osteoporosis (16) and Alzheimer's disease (17). Several studies also reveal that trehalose is capable of governing hyperglycemia in diabetes mellitus (DM) patients by alleviating impaired glucose tolerance, mitigating insulin resistance, and relieving post-meal insulin bursts (12). Moreover, Casey et al. (18) further find that trehalose is the only landmark metabolite in carbohydrate metabolism associated with DM after adjusting for fasting glucose. Based on the above evidence, we hypothesize that trehalose may be helpful to inhibit the development of DR by neuroprotection and governing hyperglycemia.

Meanwhile, the adverse effect of glutamate on the evolution of DR has been reported in multiple pieces of research. Cheng et al. (19) have observed a consistent association of insulin resistance traits with the elevation of plasma glutamate in the Framingham Heart Study and the Malmö Diet and Cancer Study. It is further demonstrated as a potentially valuable biomarker for screening DR from type 2 diabetic (T2D) patients in another metabolomic research (20). However, the intake of dietary glutamate does not affect DR occurrence according to a Korea National Diabetes Program cohort study (21). Furthermore, existing evidence suggests that glutamate excitotoxicity has a pivotal role in the pathogenesis of retinal neurodegeneration, which is one of the symbolic characteristics during the early transformation of DR (7).
Although all the above evidence suggests that both trehalose and glutamate may be related to DR, the epidemiological evidence of the trehalose role and its joint effect with glutamate on DR remains indistinct. In this two-center, propensity score matching-based case-control study, we devoted to comprehensively investigate the individual and joint effects of them on the evolution of DR using data from a serum metabolomics study.

\section{Materials and methods}

\section{Study population}

The study population was enrolled from the two affiliated hospitals of Wenzhou medical university and Anhui medical university in China from August 2017 to June 2018. Details of study design, study participants enrollment and data collection have been reported previously (22, 23). Briefly, without a history of any relevant diseases (i.e. other eye diseases, type 1 diabetes, cardiovascular disease, heart failure, cancer, infectious disease and other chronic systemic diseases), 950 participants including 755 health controls and 195 T2D patients over 35 years old were diagnosed following the diagnostic criteria recommended by World Health Organization (WHO) in 1999 and enrolled in the current study.

Fundus photographs were graded preliminarily for the level of DR and other fundus lesions by two graders depending on the retinal photographs assessment (24). If one eye was unavailable for the classification, the other one was graded. DR grading and its severity were determined depending on the worse eye, and each eye was assessed as follows: no apparent DR and any DR, which would be further classified into mild, moderate and severe non-proliferative DR (NPDR) as well as proliferative DR (PDR) according to International Clinical Diabetic Retinopathy Disease Severity Scale (24). Details of the criteria could be seen in Appendix A. Among 195 enrolled T2D patients, 83 finally were diagnosed with DR and 112 without DR (DM). The protocol was carefully prepared in accordance with Helsinki protocols and had been approved by the Ethics Committee of the Eye Hospital of Wenzhou Medical University (Number: KYK [2017] 46). Writing informed consent has been obtained from each patient after a full explanation of the purpose and nature of all procedures used.

\section{Propensity-score matching approach}

As an important chronic non-communicable disease, the likelihood of developing DR would be affected by a lot of

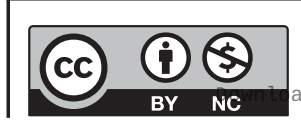

This work is licensed under a Creative Commons Attribution-NonCommercial 4.0 International License. ded from Bioscientifica.com at 04/26/2023 02:19:44PM 
factors including dyslipidemia, hypertension, metabolic syndrome, lifestyle and others. All these known or unknown confounders would modify the results and their influences should be carefully assessed before the following data analyses. However, apparent multicollinearity would inevitably occur if they were simply included in a multiple regression model because of the correlations to each other, which would highly increase the probability of overfitting and lead to incredible conclusions (25). To greatly decrease the impacts due to these known or unknown confounders and improve the robustness and credibility of our findings (26), all cases (T2D with DR) and controls (T2D without DR) were matched at a ratio of 1:1 using a propensity score matching (PSM) method, a progressively more employed strategy to control confounding bias in many observational studies during the past several decades (27). Specifically, potential confounding factors including age, gender, BMI and $\mathrm{HbA} 1 \mathrm{c}$ were fit into a multivariable logistic model with the nearest neighbor algorithm. At last, 69 pairs of cases and controls were included in the study. Further, 69 health controls were matched with T2DM patients as the blank control group based on age, gender, and BMI (Supplementary Fig. 1, see section on supplementary materials given at the end of this article).

\section{Covariates}

Associated covariatesincluded the following characteristics: demographic variables, clinical manifestation and laboratory assessments. Among them, demographic variables included age, gender, height, weight, duration of diabetes, history of hypertension, tobacco smoking and alcohol consumption, hypoglycemic therapy history, family history of diabetes, occupation as well as life habits, which were collected by a face-to-face interview using standardized questionnaires. BMI was calculated as weight $(\mathrm{kg})$ divided by height square $\left(\mathrm{m}^{2}\right)$. Clinical manifestations mainly contained SBP and diastolic blood pressure (DBP). Laboratory assessments included fasting blood glucose, HbA1c, total cholesterol, triglyceride (TG), HDL and LDL. All data collections were conducted strictly following the corresponding standardized operation procedures of this study.

\section{Serum trehalose and glutamate determination}

Based on a widely targeted metabolomics study (23), serum trehalose and glutamate were detected by experienced technicians using an ultra-performance liquid chromatography-tandem mass spectrometry system in the central laboratory of Metware Inc, a professional metabolomics institution in Wuhan City, China. The process of metabolic profiling could be found in Appendix B or our previous works $(22,23)$. To remove the batch effect on the data, quality control (QC) samples were utilized to carefully correct signal drift. Furthermore, the coefficient of variation of QC samples was used to evaluate the reliability of metabolites by a threshold of $30 \%$.

\section{Statistical analysis}

\section{Data preparation for analytics}

Poor quality of data will inevitably lead to biased and incredible decisions. So, sufficient and appropriate data pre-processing approaches should be performed before following data analyzes since they will highly improve the data quality and has been proven to play a crucial role in achieving robust and credible findings (28). To improve the data quality as much as possible, we firstly checked the completeness of covariates in terms of profiling of the number and pattern of missing values. Only those variables with a missing percentage under $30 \%$ and whose values were missed at random were imputed five times with the multiple imputations of chained equations method. In addition, we also applied other methods involved in data preparation, containing checking the values of categorical and interval variables, distribution profiling and necessary data transformation (log-transformation and Pareto scaling approaches), to make our data more suitable for analytics.

\section{Descriptive statistics of the study participants}

Continuous data with normal distribution, determined by the ShapiroWilk test, were described with mean \pm standard deviation. If their distributions were obviously skewed, they would be presented as median (the first quartile, the third quartile). Categorical variables were described as frequency (percentage). The comparability of each covariate between DR and DM was evaluated by paired t-test, Wilcoxon signed-rank test or McNemar-Bowker test as appropriate.

\section{Individual association of trehalose and glutamate with DR}

The importance of glutamate and trehalose concentrations on DR was separately examined by paired t-test, fold change (FC), and the area under receiver operating characteristic curve (AUC) via univariate conditional logistic regression

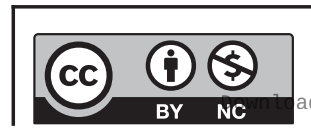

This work is licensed under a Creative Commons Attribution-NonCommercial 4.0 International License. ded from Bioscientifica.com at 04/26/2023 02:19:44PM 
model. Meanwhile, the individual association between serum trehalose and glutamate with the odds of DR was separately estimated by odds ratios (ORs) and associated 95\% CIs in the following two ways: with the exposure as a continuous variable (scaled to interquartile range (IQR)) and categorical one (quartiles) (29). Briefly, these individual associations were comprehensively quantified in three manners: (i) without adjusting for any potential confounders (Model 1); (ii) adjusting for major confounding factors including SBP and duration of diabetes (Model 2); (iii) adjusting for confounders screened by the least absolute shrinkage and selection operator (LASSO) regression model (Model 3), in which the minimum ( $\lambda \mathrm{min}$ ) criteria were applied to select the most valuable confounders (Supplementary Fig. 2). Meanwhile, the LASSO regression model with 10 -fold cross-validation was further applied to shrink the potential collinearity of confounders and internal validation (30). The likelihood of the nonlinear relationships between the two exposures and the odds of DR were additionally examined by the restricted cubic spline regression model with knots separately placed at the 5th, 50th and 95th centiles (Fig. $1)$, in which the non-linearity was tested by the likelihood ratio test depending on the comparison between the two models with the linear term only or with a combination of the linear and cubic spline terms (29).

\section{Interaction of trehalose and glutamate on DR}

For reasonable evaluation of the joint effects of serum trehalose and glutamate, they were both split into binary categories according to each median. The interaction of trehalose with glutamate on DR was assessed on a multiplicative scale using OR (95\% CI) and its significance was tested via the Wald test. In addition, the additivity of effect in the two exposures was also examined by modeling the relative risk due to interaction (RERI) using variance estimates recovery method (31), in which a RERI of 0 indicated no additive interaction existed.

\section{Sensitivity analysis}

We also performed the following sensitivity analyses to ensure the robustness and credibility of our results: (i) we repeated all above analyses utilizing the dataset without multiple imputations to testify the influence of imputation on the results; (ii) dietary favor variables including salt consumption, prefer high oil diet or not, frequency of the intakes of picked food, vegetables, fish and others were additionally included in the models to adjust for potential impacts due to diet on the association of the two exposures with the likelihood of developing DR; (iii) all analyses were additionally repeated only in the NPDR patients and their matched controls to assess the consistency of our findings; (iv) we further repeated analyses between DR subjects and their corresponding health control subjects to evaluate whether the conclusion could be generalized to healthy people and DR patients.

Both data management and statistical analyses were performed using STATA MP (version 15.1, (C) 1985-2017 StataCorp LLC, College Station, Texas, USA) and R (version 4.0.5, R Foundation). The 'glmnet' package in $\mathrm{R}$ was used to
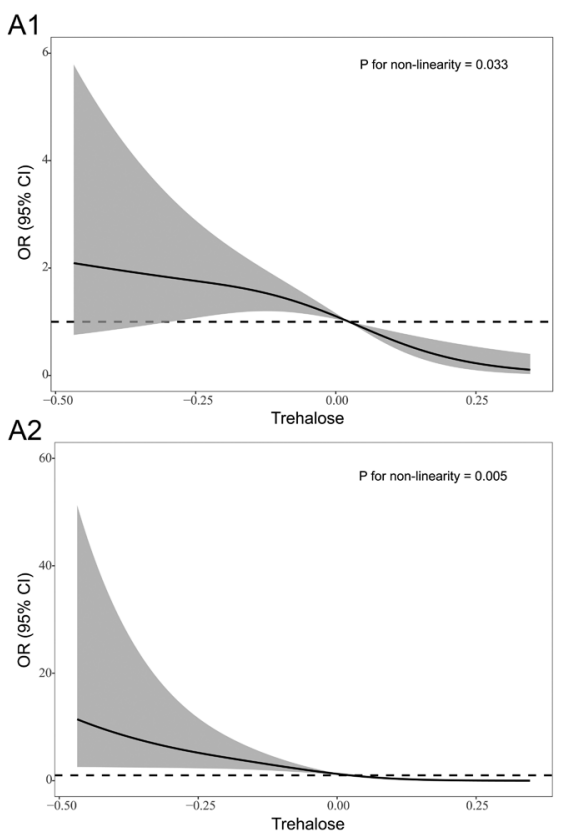

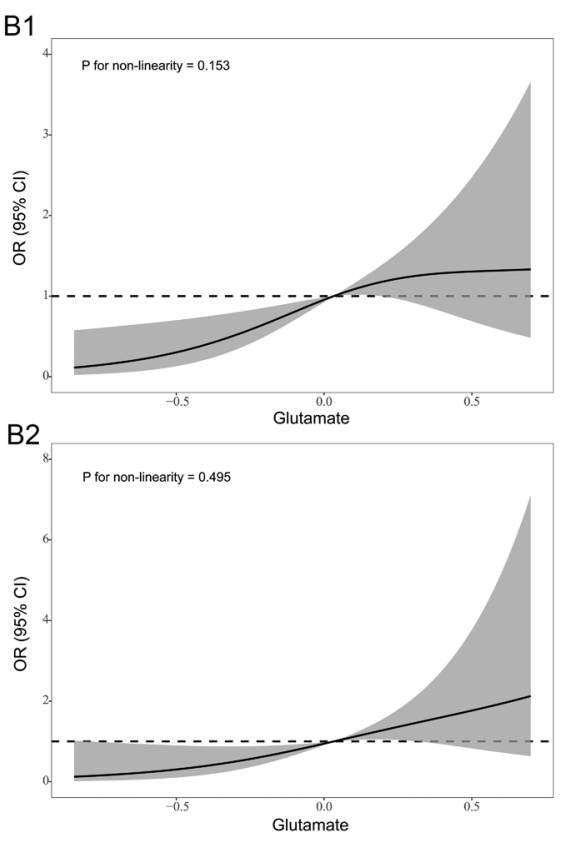

\section{Figure 1}

Associations of standard-transformed trehalose (A) and glutamate (B) with the odds of diabetic retinopathy. (A1 and B1) adjusting for SBP and duration of diabetes; (A2 and B2) Adjusting for confounders screened by the LASSO regression model. Odds ratios and $95 \% \mathrm{Cls}$ are indicated by solid lines and shaded areas, respectively. The intersection point of the solid line and dash line is the median of each metabolite, with knots of restricted cubic spline logistic regression model placed at 5th, 50th and 95th percentiles. https://ec.bioscientifica.com https://doi.org/10.1530/EC-21-0474 (c) 2022 The authors Published by Bioscientifica Ltd

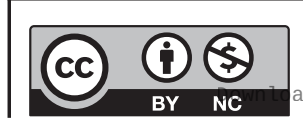

This work is licensed under a Creative Commons Attribution-NonCommercial 4.0 International License. ded from Bioscientifica.com at 04/26/2023 02:19:44PM via free access 
perform the least absolute shrinkage and selection operator (LASSO) regression. The 'ggplot2' and 'rms' packages were applied to generate a restricted cubic spline regression model. The 'epiR' package was utilized to evaluate the additive interaction of trehalose and glutamate. All tests were two-sided and $P$-value $\leq 0.05$ was considered statistically significant.

\section{Results}

\section{Characteristics of study participants}

Among 69 cases, the numbers of mild, moderate and severe NPDRs as well as PDR patients were 9, 31, 20 and 9 , respectively. Table 1 showed the demographic, clinical and laboratory characteristics of the 138 participants. In brief, the median (first quartile, third quartile) of age was $55(49,63)$ years. A majority $(53.6 \%)$ of the subjects were men, more than $60 \%$ came from the Wenzhou area, $93.8 \%$ were married and $22.9 \%$ were over the high school level of education. As compared to DM, DR patients were more likely to be older $(P$-value $=0.022)$, to have higher SBP $(P$-value $=0.003)$, longer duration of diabetes $(P$-value $=0.002)$ and higher proportion to receive treatments $(P$-value $<0.001)$. Other demographic, clinical and laboratory features were not significantly different between DR and DM.

Twenty-two baseline variables (Table 1) were included in the LASSO regression and ten variables were selected as important confounders, including TG, SBP, DBP, duration of diabetes, residence, degree of education, smoking status, history of hypertension, insulin therapy immediately after diagnosis of DM or not (ITIADM) and therapy or not (Supplementary Fig. 2).

Table 1 Baseline characteristics among participants.

\begin{tabular}{l}
\hline Characteristics \\
\hline Continuous characteristics \\
Age (years) \\
BMI $\left(\mathrm{kg} / \mathrm{m}^{2}\right)$ \\
FPG $(\mathrm{mmol} / \mathrm{L})$ \\
HbA1c $(\%)$ \\
LDL $(\mathrm{mmol} / \mathrm{L})$ \\
HDL (mmol/L) \\
TG (mmol/L) \\
TC (mmol/L) \\
SBP (mmHg) \\
DBP (mm Hg) \\
Duration of diabetes, years) \\
Category characteristics, $n$ (\%) \\
Men \\
Wenzhou (or Hefei) \\
Married \\
High school and above \\
Occupation \\
Manual workers \\
Mental worker \\
Both \\
History of hypertension \\
Smoking status \\
Non-smokers \\
Ex-smokers \\
Current smokers \\
Drinking status \\
Non-drinkers \\
Ex-drinkers \\
Current drinkers \\
Family history of diabetes \\
Hypoglycemic therapy \\
ITIADM \\
\hline \\
Man
\end{tabular}

\begin{tabular}{c}
\hline Pooled $(n=138)$ \\
\hline $55.0(49.0,63.0)$ \\
$24.5 \pm 3.4$ \\
$8.4(6.7,10.9)$ \\
$10.0 \pm 2.1$ \\
$2.6 \pm 1.0$ \\
$1.1(0.8,1.3)$ \\
$1.5(1.0,2.1)$ \\
$4.6 \pm 1.3$ \\
$130.0(120.0,144.0)$ \\
$78.0(72.0,86.0)$ \\
$10.0(5.0,15.0)$ \\
$74(53.6)$ \\
$84(60.9)$ \\
$121(93.8)$ \\
$30(22.9)$ \\
$65(50.4)$ \\
$26(20.2)$ \\
$38(29.5)$ \\
$47(35.9)$ \\
$77(58.8)$ \\
$14(10.7)$ \\
$40(30.5)$ \\
$62(47.3)$ \\
$12(9.2)$ \\
$57(43.5)$ \\
$67(51.9)$ \\
$106(87.6)$ \\
$29(22.3)$ \\
\\
\\
\end{tabular}

\begin{tabular}{c}
\hline T2DM $(n=69)$ \\
\hline $53.0(48.0,61.0)$ \\
$24.4 \pm 3.2$ \\
$8.4(6.9,12.0)$ \\
$10.1 \pm 2.3$ \\
$2.7 \pm 1.0$ \\
$1.0(0.8,1.3)$ \\
$1.6(1.0,2.2)$ \\
$4.7 \pm 1.1$ \\
$124.0(118.0,139.0)$ \\
$79.0(74.0,86.0)$ \\
$8.0(4.0,13.0)$
\end{tabular}

\begin{tabular}{c}
\hline $\mathbf{D R}(n=69)$ \\
\hline $56.0(51.0,65.0)$ \\
$24.6 \pm 3.5$ \\
$8.5(6.3,10.2)$ \\
$9.9 \pm 1.9$ \\
$2.6 \pm 1.1$ \\
$1.1(0.9,1.3)$ \\
$1.4(1.0,1.9)$ \\
$4.5 \pm 1.4$ \\
$135.0(122.0,148.0)$ \\
$76.0(70.0,85.0)$ \\
$12.0(8.0,17.0)$
\end{tabular}

P value

0.022

0.773

0.225

0.500

0.617

0.703

0.184

0.337

0.003

0.449

0.002

$38(55.1)$

$36(52.2)$

$61(95.3)$

$18(27.3)$

31 (47.7)

15 (23.1)

19 (29.2)

$19(28.8)$

41 (62.1)

$6(9.1)$

19 (28.8)

$33(50.0)$

3 (4.5)

30 (45.5)

30 (47.6)

44 (75.9)

$19(29.2)$

\begin{tabular}{rr}
$36(52.2)$ & 0.625 \\
$48(69.6)$ & 0.074 \\
$60(92.3)$ & 0.727 \\
$12(18.5)$ & 0.189 \\
& 0.825 \\
$34(53.1)$ & \\
$11(17.2)$ & \\
$19(29.7)$ & \\
$28(43.1)$ & 0.078 \\
$36(55.4)$ & 0.530 \\
$8(12.3)$ & \\
$21(32.3)$ & \\
& \\
$29(44.6)$ & 0.921 \\
$9(13.8)$ & \\
$27(41.5)$ & \\
$37(56.1)$ & 0.556 \\
$62(98.4)$ & $<0.001$ \\
$10(15.4)$ & 0.093 \\
\hline
\end{tabular}

DBP, diastolic blood pressure; DR, type 2 diabetes mellitus with diabetic retinopathy; FPG, fasting plasma glucose; HbA1C, glycated hemoglobin; ITIADM, insulin therapy immediately after diagnosis of DM or not; LDL, low density lipoprotein; HDL, high density lipoprotein; SBP, systolic blood pressure; TC, total cholesterol; TG, triglyceride; T2DM, type 2 diabetes mellitus without diabetic retinopathy; Family history of diabetes, any of the patient's siblings, parents or grandparents has a history of diabetes.

https://ec.bioscientifica.com https://doi.org/10.1530/EC-21-0474
(C) 2022 The authors Published by Bioscientifica Ltd

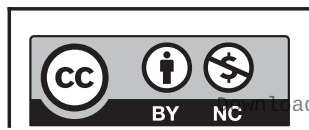

This work is licensed under a Creative Commons Attribution-NonCommercial 4.0 International License. ded from Bioscientifica com at 04/26/2023 02:19:44PM via free access 


\section{Individual association of trehalose and glutamate} with DR

As could be seen in Table 2, DR patients tended to have lower serum trehalose $(\mathrm{FC}=0.89, P$-value $<0.001))$ and higher serum glutamate $(\mathrm{FC}=1.18, \quad P$-value $=0.007)$ when comparing with their counterparts. In addition, the two exposures were also observed to have moderate discrimination abilities when separately using them as potential biomarkers in distinguishing DR from T2D patients $\left(\mathrm{AUC}_{\text {trehalose }}=0.70, \mathrm{AUC}_{\text {glutamate }}=0.65\right)$.

The individual associations of serum trehalose and glutamate with the odds of DR were summarized in Table 3. After adjusting for the 10 confounders screened by LASSO regression model (Supplementary Fig. 2), the likelihood of DR was still significantly and independently associated with the two exposures. With per IQR elevation of serum trehalose, the adjusted odds of DR averagely decreased by $86 \%$ (OR: 0.14 ; 95\% CI: $0.06,0.33$ ). When comparing with participants in the lowest quartile, adjusted odds of DR in subjects with the second, third and fourth quartiles trehalose apparently decreased by 80,86 and 99\%, respectively. However, the adjusted likelihood to get DR averagely increased by $90 \%$ (OR: $1.90 ; 95 \%$ CI: $1.20,3.00)$ with per IQR rise of serum glutamate. When comparing with subjects in the first quartile of glutamate, the adjusted odds of developing DR for participants in the highest quartile significantly increased by $459 \%$ (OR: 5.59; 95\% CI: 1.51, 20.62). Meaningful trends were detected in both glutamate and trehalose. To detect whether the trends were linear, the restricted cubic spline logistic regression models were performed after adjusting confounders (Fig. 1). A negative nonlinear association was detected between trehalose and the odds of DR ( $P$ for nonlinearity $<0.05)$, while a positive linear association was also found between glutamate and the odds of DR ( $P$ for non-linearity > 0.05)

\section{Joint associations between serum trehalose and glutamate on DR}

As we could see in Table 4, the likelihood of developing DR was significantly increased with exposure to lower serum trehalose and higher glutamate. The highest effects (adjusted OR: 36.81; 95\% CI: 6.75, 200.61) were detected among T2D patients whose serum trehalose was in the low category (trehalose < median) and serum glutamate was in the upper 50th percentile after adjusting LASSO-selected confounders (Fig. 2). Interestingly, compared to those who were exposed to both high trehalose and low glutamate, the odds of DR were not significantly changed among participants exposed to either low serum trehalose or high serum glutamate alone, which strongly indicated that their associations with DR would be largely affected by each other and the interaction effect of the two exposures on DR should be carefully investigated. The results demonstrated that serum trehalose and glutamate were jointly associated with DR, and the multiplicative interaction term was significant $(P$-value $=0.001)$. RERI estimated for the relative excess risk due to interaction of low trehalose and high glutamate on DR exceeded 0 (RERI $=8.35)$. However, its $95 \%$ CI ( -22.49 to 79.18$)$ included 0 , which indicated that RERI was not significant (Table 4).

\section{Sensitivity analysis}

The individual and joint associations between serum trehalose and glutamate with the odds of DR were remained consistent in participants without missing values imputation (Supplementary Table 1), with additional adjusting for dietary favor (Supplementary Table 2) and in NPDR patients only (Supplementary Table 3), Interestingly, the association between trehalose and DR was disappeared after taking health control group as a reference and adjusting HbA1c (Supplementary Table 4). Furthermore, the multiplicative interaction of trehalose and glutamate with DR was not significant between DR and health controls (Supplementary Table 4). The boxplots of trehalose and glutamate among three groups were summarized in Supplementary Fig. 3 for explaining this phenomenon. In general, the associations were basically consistent with the main results which ensured the robustness of our findings.

\section{Discussion}

To the best of our knowledge, this is the first report to thoroughly quantify the joint association of serum

Table 2 Distinct metabolites in participants with diabetic retinopathy and their counterparts.

\begin{tabular}{|c|c|c|}
\hline Metabolites & Adduction & $\mathbf{R T}(\min )$ \\
\hline Trehalose & {$[\mathrm{M}-\mathrm{H}]-$} & 0.72 \\
\hline Glutamate & {$[\mathrm{M}-\mathrm{H}]-$} & 0.78 \\
\hline
\end{tabular}

\begin{tabular}{c}
\hline $\mathbf{m} / \mathbf{z}$ \\
\hline 342.116 \\
147.053 \\
\hline
\end{tabular}

$\begin{array}{r}\hline \text { P value } \\ \hline<0.001 \\ 0.007 \\ \hline\end{array}$

\begin{tabular}{c}
\hline $\mathbf{F C}$ \\
\hline 0.89 \\
1.18 \\
\hline
\end{tabular}

\begin{tabular}{l} 
AUC \\
\hline 0.70 \\
0.65
\end{tabular}

AUC, area under the curve; FC, fold change; $\mathrm{m} / \mathrm{z}$, actual mass-to-charge ratio; RT, retention time; VIP, variable importance in the project.

https://ec.bioscientifica.com https://doi.org/10.1530/EC-21-0474 (c) 2022 The authors Published by Bioscientifica Ltd

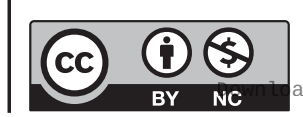

This work is licensed under a Creative Commons Attribution-NonCommercial 4.0 International License. ded from Bioscientifica.com at 04/26/2023 02:19:44PM 
Table 3 Individual relationship of trehalose and glutamate with the odds of diabetic retinopathy.

\begin{tabular}{|c|c|c|c|c|c|c|c|c|}
\hline \multirow[b]{2}{*}{ Metabolites } & \multirow[b]{2}{*}{$N$} & \multirow[b]{2}{*}{ Cases (\%) } & \multicolumn{2}{|c|}{ Model 1} & \multicolumn{2}{|l|}{ Model 2} & \multicolumn{2}{|l|}{ Model 3} \\
\hline & & & OR $(95 \% \mathrm{Cl})$ & $P$ & OR $(95 \% \mathrm{Cl})$ & $P$ & OR $(95 \% \mathrm{Cl})$ & $P$ \\
\hline \multicolumn{9}{|l|}{ Trehalose } \\
\hline Per IQR & & & $0.41(0.26,0.65)$ & $<0.001$ & $0.40(0.25,0.65)$ & $<0.001$ & $0.14(0.06,0.33)$ & $<0.001$ \\
\hline \multicolumn{9}{|l|}{ Quartiles } \\
\hline $\mathrm{Q}_{1}$ & 34 & $23(67.60)$ & $1.00(1.00,1.00)$ & Ref. & $1.00(1.00,1.00)$ & Ref. & $1.00(1.00,1.00)$ & Ref. \\
\hline $\mathrm{Q}_{2}$ & 35 & $19(54.30)$ & $0.57(0.21,1.51)$ & 0.257 & $0.44(0.15,1.27)$ & 0.129 & $0.20(0.05,0.83)$ & 0.027 \\
\hline $\mathrm{Q}_{3}$ & 35 & $21(60.00)$ & $0.72(0.27,1.92)$ & 0.509 & $0.70(0.25,1.98)$ & 0.500 & $0.14(0.03,0.63)$ & 0.011 \\
\hline $\mathrm{Q}_{4}$ & 34 & $6(17.60)$ & $0.10(0.03,0.32)$ & $<0.001$ & $0.09(0.03,0.31)$ & $<0.001$ & $0.01(<0.01,0.05)$ & $<0.001$ \\
\hline$P$ for trend & & & & $<0.001$ & & 0.001 & & $<0.001$ \\
\hline \multicolumn{9}{|l|}{ Glutamate } \\
\hline Per IQR & & & $1.62(1.15,2.30)$ & 0.006 & $1.73(1.19,2.52)$ & 0.004 & $1.90(1.20,3.00)$ & 0.006 \\
\hline \multicolumn{9}{|l|}{ Quartiles } \\
\hline $\mathrm{Q}_{1}$ & 34 & $11(32.40)$ & $1.00(1.00,1.00)$ & Ref. & $1.00(1.00,1.00)$ & Ref. & $1.00(1.00,1.00)$ & Ref. \\
\hline $\mathrm{Q}_{2}$ & 35 & $16(45.70)$ & $1.76(0.66,4.69)$ & 0.257 & $1.68(0.58,4.85)$ & 0.339 & $1.43(0.42,4.93)$ & 0.571 \\
\hline $\mathrm{Q}_{3}$ & 35 & $21(60.00)$ & $3.14(1.17,8.41)$ & 0.023 & $3.11(1.07,9.00)$ & 0.037 & $3.00(0.81,11.16)$ & 0.101 \\
\hline $\mathrm{Q}_{4}$ & 34 & $21(61.80)$ & $3.38(1.25,9.16)$ & 0.017 & $4.55(1.53,13.55)$ & 0.007 & $5.59(1.51,20.62)$ & 0.010 \\
\hline$P$ for trend & & & & 0.008 & & 0.003 & & 0.004 \\
\hline
\end{tabular}

Model 1: unadjusted. Model 2: adjusted for SBP and duration of diabetes. Model 3: adjusted for confounders screened by the LASSO regression model, including TG, SBP, DBP, duration of diabetes, residence, degree of education, smoking status, history of hypertension, ITIADM and therapy or not. IQR, interquartile range; OR, odds ratio; Q1, the first quartile; Q2, the second quartile; Q3, the third quartile; Q4, the fourth quartile.

trehaloseand glutamatewith theodds ofDR. After adjusting for confounders screened by LASSO models, T2D patients with both low serum trehalose (trehalose $<$ median) and high serum glutamate (glutamate $\geq$ median) were more than 30 times as likely to be DR than those with high serum trehalose and low serum glutamate. Interaction on the multiplicative scale achieved a significant level, whereas the additive interaction was not significant at the same time. In addition, our results also revealed that T2D patients with either low serum trehalose or high serum glutamate alone were not at significantly higher risk of developing DR than those whose serum trehalose over median and glutamate below the 50th percentile. Our findings distinctly suggest that trehalose supplement and decreasing glutamate not only are independent protective factors for DR but also synergistically decrease the likelihood of developing DR.

\section{Comparison with other studies and biological plausibility}

Increasing evidence suggests that the risk of DR is affected by several elements including metabolism, age, gender, BMI, dietary habits, duration of diabetes, hyperglycemia, hypertension, and dyslipidemia $(1,3,4,32)$. To control the influence of age, gender, BMI and HbA1c on the findings and increase the robustness of our results, we conducted this propensity score matching-based case-control study. A LASSO regression model was utilized to screen appropriate confounding factors to be controlled. At last, confounders including the duration of diabetes, hyperglycemia, hypertension, dyslipidemia and others were adjusted when quantifying the association of serum trehalose and glutamate with DR by multiple conditional logistic regression as well as restricted cubic spline regression

Table 4 Joint effect of trehalose and glutamate on diabetic retinopathy.

\begin{tabular}{|c|c|c|c|c|c|c|c|c|c|}
\hline \multirow{2}{*}{\multicolumn{2}{|c|}{ Metabolite > median }} & \multirow[b]{2}{*}{$\boldsymbol{N}$} & \multirow[b]{2}{*}{ Cases $(\%)$} & \multicolumn{2}{|l|}{ Model 1} & \multicolumn{2}{|l|}{ Model 2} & \multicolumn{2}{|l|}{ Model 3} \\
\hline & & & & OR $(95 \% \mathrm{Cl})$ & $P$ & OR $(95 \% \mathrm{Cl})$ & $P$ & OR $(95 \% \mathrm{Cl})$ & $P$ \\
\hline \multicolumn{10}{|c|}{ Trehalose Glutamate } \\
\hline Yes & No & 32 & $13(40.60)$ & $1.00(1.00$ to 1.00$)$ & Ref. & $1.00(1.00$ to 1.00$)$ & Ref. & $1.00(1.00$ to 1.00$)$ & Ref. \\
\hline No & No & 37 & $14(37.80)$ & $0.89(0.34$ to 2.35$)$ & 0.813 & $0.68(0.23$ to 1.98$)$ & 0.478 & $1.32(0.33$ to 5.26$)$ & 0.689 \\
\hline Yes & Yes & 37 & $14(37.80)$ & $0.89(0.34$ to 2.35$)$ & 0.813 & $0.86(0.30$ to 2.43$)$ & 0.771 & $0.71(0.21$ to 2.39$)$ & 0.579 \\
\hline No & Yes & 32 & $28(87.50)$ & 10.23 (2.89 to 36.18$)$ & $<0.001$ & 11.43 (3.04 to 43.05$)$ & $<0.001$ & $36.81(6.75$ to 200.61$)$ & $<0.001$ \\
\hline \multicolumn{4}{|c|}{ Interaction (Glutamate*Trehalose) } & 12.93 (2.68 to 62.40$)$ & 0.001 & 19.65 (3.58 to 107.87$)$ & 0.001 & $39.21(4.75$ to 323.41$)$ & 0.001 \\
\hline \multicolumn{4}{|c|}{ RERI $(95 \% \mathrm{Cl})$} & $9.45(-2.84$ to 21.74$)$ & & $10.90(-3.66$ to 25.45$)$ & & $8.35(-22.49$ to 79.18$)$ & \\
\hline
\end{tabular}

Model 1: unadjusted. Model 2: adjusted for SBP and duration of diabetes. Model 3: adjust for confounders screened by the LASSO regression model, including TG, SBP, DBP, duration of diabetes, residence, degree of education, smoking status, history of hypertension, ITIADM and therapy or not. $I Q R$, interquartile range; RERI, relative excess risk of interaction; OR, odds ratio.

https://ec.bioscientifica.com https://doi.org/10.1530/EC-21-0474
(C) 2022 The authors Published by Bioscientifica Ltd

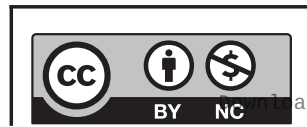

This work is licensed under a Creative Commons Attribution-NonCommercial 4.0 International License. ded from Bioscientifica.com at $04 / 26 / 2023$ 02:19:44PM 


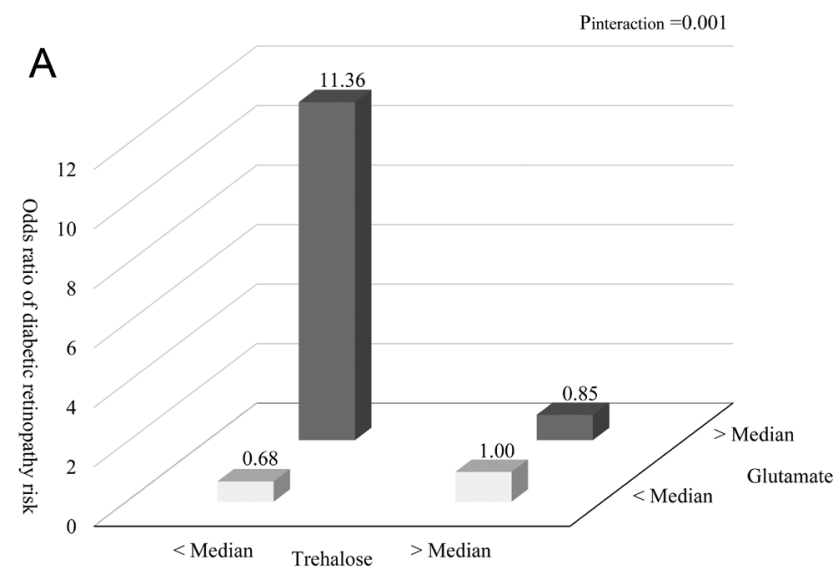

B

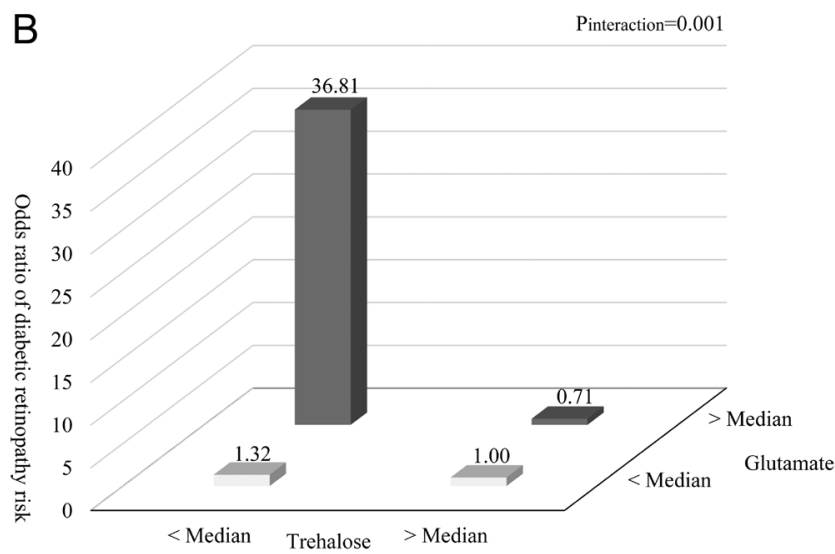

Figure 2

Joint associations between trehalose and glutamate with the odds of diabetic retinopathy. (A) adjusting for systolic blood pressure, duration of diabetes; and (B) adjusting for confounders screened by the LASSO regression model.

models. In addition, a series of sensitivity analyses were further performed to ensure the robustness of our findings. All results strongly indicated that the individual and joint associations of the two exposures with DR were robust and credible.

Previous evidence on serum trehalose concerning the incidence of DR is scarce, especially in humans. Fortunately, its effects on glucose regulation and neuroprotection have been found in both in vitro and in vivo (12). Arial et al. have reported that trehalose intake suppresses mesenteric adipocyte hypertrophy, declines damaged glucose tolerance during the oral glucose tolerance test, and mitigates insulin resistance in both metabolic syndrome and obese mouse models $(15,33)$. Available evidence reports that TREH may highly affect trehalase activity and is related to T2DM (14, 34), TREH in DR patients may naturally inhibit trehalose activity and induce lower trehalose concentration than that of DM patients without DR. Furthermore, gut microbiota (e.g. E. coli) can resist external stresses and maintain normal cellular physiological functions by producing trehalose. Meanwhile, available evidence has also suggested the association between T2DM and intestinal flora disorders (35). Since the diabetic durations of DR patients are generally longer than that of DM patients without this specific complication, DR patients are more prone to intestinal flora disorders, which may inhibit the synthesis of trehalose in gut microbiota and the absorption of trehalose by the intestine, leading to reduced serum trehalose. Several studies report that trehalose regulation can counteract high-sugar-diet and activate hepatic fasting-like effect $(36,37)$. Recently, A randomized control study demonstrates that moderate consumption of trehalose is helpful to maintain glucose homeostasis, which plays a vital role in the process of DR, in healthy people (38). Meanwhile, available evidence has also demonstrated the potential therapeutic value of trehalose in retinal and neurodegenerative diseases $(17,39)$. Lotfi et al. (39) show that trehalose can reduce retinal degeneration and neuroinflammation by curing lysosomal storage disorders caused by lysosomal hydrolase deficiency, and potentially alleviate retinal degeneration and vision loss which is the main damage of DR. In addition, Tanaka et al. also testify that trehalose can alleviate polyglutamine-mediated pathology to improve neurodegenerative disease (17). So, we assume that the saccharide-polyglutamine interaction with trehalose may explain the strong multiplicative interaction observed in this study. Laskowska et al. (40) and Moruno Algara et al. (41) report that excess glucose may stimulate non-enzymatic glycosylation and the formation of advanced glycation end products (AGEs) bound to proteins during reduced trehalose synthesis. The inhibition of AGEs by trehalose may be a new therapeutic strategy to prevent DR occurrence since the AGEs-the receptor of the AGEs axis plays a central role in sustained inflammation, neurodegeneration, and retinal microvascular dysfunction occurring during diabetic retinopathy $(42,43,44)$. This assumption needs to be verified by further studies.

Furthermore, the role of glutamate in the progress of DR has been elucidated in some studies. As a major excitatory neurotransmitter, glutamate accumulates in DR and ultimately results in excitotoxic cell death $(7,45)$, which plays a key role in DR neurodegeneration (46) and is widely considered as the initiating factor that leads to vascular damage (47). Based on two different cohorts, Cheng et al. report that plasma glutamate is related to insulin resistance and can be applied to predict DR (20). However, the predictive value of dietary glutamate is unsatisfactory (21), which implies that plasma glutamate

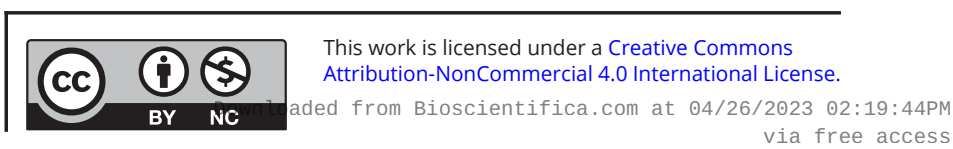


may not relate to dietary habits. Moreover, Simo et al. (7) report that increased glutamate can trigger vascular endothelial growth factor activation, which contributes to microangiopathy and results in DR. Although the exact evidence on the relationship of glutamate between retinal and serum is not yet available, we can still conclude that high level of serum glutamate is an independent risk factor of DR and closely related to its rapid development.

\section{Strengths and limitations}

There are several strengths in our study. First, the participants were enrolled from both outpatients and inpatients in the Endocrinology Department of two top, huge hospitals in Zhejiang and Anhui provinces, which cover more than 120 million people in China. In addition, four types of DR patients (mild NPDR, moderate NPDR, severe NPDR and PDR) could be found in this study. This would be very helpful to reduce selection bias to a large extent. Second, the PSM approach was used to achieve more comparable cases and controls. It would well balance the impacts due to some important confounders and make our findings more robust and credible. Third, careful data preparation was conducted before the behind analyses to improve the quality of our data. Meanwhile, potential overfitting and multicollinearity were well-considered when comprehensively quantifying the individual and joint association of serum trehalose and glutamate with DR by adjusting for the confounders screened via LASSO regression models. Fourth, necessary sensitivity analyses were conducted to ensure the robustness and reliability of our findings and make conclusions that can be extended to the population between healthy people and DR patients.

However, our study also has limitations. First, our findings are mainly based on a case-control study and need to be confirmed by longitudinal or experimental studies. Second, potential recall bias or reporting bias might exist in some covariates (dietary habits, etc.) obtained via questionnaire and affected our results to some extent. Third, this was not a large sample size-based study restricted by the actual situation, but the power was still over 0.9 which had been carefully evaluated in our previous work (23). Fourth, although the participants were enrolled from two centers, our findings might not be perfectly generalized to other populations.

In summary, our work revealed that T2D patients individually and jointly exposed to high trehalose and low glutamate would like to have lower odds of DR, especially in those with both high level of trehalose and low level of glutamate at the same time. We reported for the first time that there was an apparent synergistic effect between serum trehalose and glutamate on the initiation and development of DR. Our findings strongly indicate that retinal neurodegeneration plays an important role in the pathogenesis of DR and provide insights into the potential value for proposing new treatment target. Longitudinal studies are required to confirm our findings.

\section{Supplementary materials}

This is linked to the online version of the paper at https://doi.org/10.1530/ EC-21-0474.

\section{Declaration of Interest}

The authors declare that there is no conflict of interest that could be perceived as prejudicing the impartiality of the research reported.

\section{Funding}

This work was supported by National Nature Science Foundation of China (82070833), Zhejiang University Student Science and Technology Innovation Activity Plan (2021R413075), Zhejiang Basic Public Welfare Research Project (LGF19H260011) and Major Project of the Eye Hospital of Wenzhou Medical University (YNZD201602). Part of this work was also funded by Zhejiang Provincial Key Research and Development Program (2021C03070) and Natural Science Foundation of Zhejiang Province (LZ19H020001).

\section{Author contribution statement}

Chengnan Guo: Conceptualization, Data analysis, Methodology, Writing original draft, Writing - review and editing. Yixi Xu: Data analysis, Writing - review and editing, Supervision. Shuzhen Zhao, Huihui Li, Dongzhen Jin, Xin Xu, Fang Peng, Zhezheng Xia, Mingzhu Che, Mengyuan Lai and Ruogu Huang: Contributed to the epidemiological investigation, sample handling, data management and analysis. Depeng Jiang and Chao Zheng: Writing - review and editing. Guangyun Mao: Conceptualization, Funding acquisition, Supervision, Writing - review and editing.

\section{Acknowledgements}

The author would like to thank all the participants of the studies and the colleagues from Wenzhou Medical University for their hard works and collaborations. The authors thank Ms Xue-Yi Zhang (Wuhan Institute of Virology, CAS) for their kind assistance. The author would also thank SpecAlly Life Technology Co., Ltd. and Mr Meng Gao and Wuhan Metware Biotechnology Co., Ltd. for their contributions and assistance in this study.

\section{References}

1 Cheung N, Mitchell P \& Wong TY. Diabetic retinopathy. Lancet 2010 376 124-136. (https://doi.org/10.1016/S0140-6736(09)62124-3)

2 International Diabetes Federation. IDF Diabetes Atlas, 9th ed.. Brussels, Belgium: IDF, 2019.

3 Vujosevic S, Aldington SJ, Silva P, Hernández C, Scanlon P, Peto T \& Simó R. Screening for diabetic retinopathy: new perspectives and 
challenges. Lancet: Diabetes and Endocrinology $20208337-347$. (https:// doi.org/10.1016/S2213-8587(19)30411-5)

4 Wong TY, Cheung CM, Larsen M, Sharma S \& Simo R. Diabetic retinopathy. Nature Reviews: Disease Primers 20162 16012. (https://doi. org/10.1038/nrdp.2016.12)

5 Yau JW, Rogers SL, Kawasaki R, Lamoureux EL, Kowalski JW, Bek T, Chen SJ, Dekker JM, Fletcher A, Grauslund J, et al. Global prevalence and major risk factors of diabetic retinopathy. Diabetes Care 201235 556-564. (https://doi.org/10.2337/dc11-1909)

6 Sabanayagam C, Banu R, Chee ML, Lee R, Wang YX, Tan G, Jonas JB, Lamoureux EL, Cheng CY, Klein BEK, et al. Incidence and progression of diabetic retinopathy: a systematic review. Lancet: Diabetes and Endocrinology 20197 140-149. (https://doi.org/10.1016/S22138587(18)30128-1)

7 Simo R, Hernandez C \& European Consortium for the Early Treatment of Diabetic Retinopathy (EUROCONDOR). Neurodegeneration in the diabetic eye: new insights and therapeutic perspectives. Trends in Endocrinology and Metabolism 201425 23-33. (https://doi. org/10.1016/j.tem.2013.09.005)

8 Simo R, Hernandez C \& European Consortium for the Early Treatment of Diabetic Retinopathy (EUROCONDOR). Neurodegeneration is an early event in diabetic retinopathy: therapeutic implications. British Journal of Ophthalmology 201296 1285-1290. (https://doi.org/10.1136/ bjophthalmol-2012-302005)

9 Antonetti DA, Barber AJ, Bronson SK, Freeman WM, Gardner TW, Jefferson LS, Kester M, Kimball SR, Krady JK, LaNoue KF, et al. Diabetic retinopathy: seeing beyond glucose-induced microvascular disease. Diabetes 200655 2401-2411. (https://doi.org/10.2337/db05-1635)

10 Lorenzi M \& Gerhardinger C. Early cellular and molecular changes induced by diabetes in the retina. Diabetologia 200144 791-804. (https://doi.org/10.1007/s001250100544)

11 Abokyi S, Shan SW, To CH, Chan HH \& Tse DY. Autophagy upregulation by the TFEB inducer trehalose protects against oxidative damage and cell death associated with NRF2 inhibition in human RPE cells. Oxidative Medicine and Cellular Longevity 202020205296341. (https://doi.org/10.1155/2020/5296341)

12 Sokolowska E, Sadowska A, Sawicka D, Kotulska-Bablinska I \& Car H. A head-to-head comparison review of biological and toxicological studies of isomaltulose, d-tagatose, and trehalose on glycemic control. Critical Reviews in Food Science and Nutrition 2021 1-26. (https://doi.org /10.1080/10408398.2021.1895057)

13 Lee HJ, Yoon YS \& Lee SJ. Mechanism of neuroprotection by trehalose: controversy surrounding autophagy induction. Cell Death and Disease 20189 712. (https://doi.org/10.1038/s41419-018-0749-9)

14 Muller YL, Hanson RL, Knowler WC, Fleming J, Goswami J, Huang K, Traurig M, Sutherland J, Wiedrich C, Wiedrich K, et al. Identification of genetic variation that determines human trehalase activity and its association with type 2 diabetes. Human Genetics 2013132 697-707. (https://doi.org/10.1007/s00439-013-1278-3)

15 Arai C, Arai N, Mizote A, Kohno K, Iwaki K, Hanaya T, Arai S, Ushio S $\&$ Fukuda S. Trehalose prevents adipocyte hypertrophy and mitigates insulin resistance. Nutrition Research 201030 840-848. (https://doi. org/10.1016/j.nutres.2010.10.009)

16 Nishizaki Y, Yoshizane C, Toshimori Y, Arai N, Akamatsu S, Hanaya T, Arai S, Ikeda M \& Kurimoto M. Disaccharide-trehalose inhibits bone resorption in ovariectomized mice. Nutrition Research 200020 653-664. (https://doi.org/10.1016/S0271-5317(00)00155-X)

17 Tanaka M, Machida Y, Niu S, Ikeda T, Jana NR, Doi H, Kurosawa M, Nekooki M \& Nukina N. Trehalose alleviates polyglutamine-mediated pathology in a mouse model of Huntington disease. Nature Medicine 200410 148-154. (https://doi.org/10.1038/nm985)

18 Rebholz CM, Yu B, Zheng Z, Chang P, Tin A, Kottgen A, Wagenknecht LE, Coresh J, Boerwinkle E \& Selvin E. Serum metabolomic profile of incident diabetes. Diabetologia 201861 1046-1054. (https://doi.org/10.1007/s00125-018-4573-7)
19 Cheng S, Rhee EP, Larson MG, Lewis GD, McCabe EL, Shen D, Palma MJ, Roberts LD, Dejam A, Souza AL, et al. Metabolite profiling identifies pathways associated with metabolic risk in humans. Circulation 2012125 2222-2231. (https://doi.org/10.1161/ CIRCULATIONAHA.111.067827)

20 Rhee SY, Jung ES, Park HM, Jeong SJ, Kim K, Chon S, Yu SY, Woo JT \& Lee $\mathrm{CH}$. Plasma glutamine and glutamic acid are potential biomarkers for predicting diabetic retinopathy. Metabolomics 201814 89. (https:// doi.org/10.1007/s11306-018-1383-3)

21 Park SY, Kim J, Son JI, Rhee SY, Kim DY, Chon S, Lim H \& Woo JT. Dietary glutamic acid and aspartic acid as biomarkers for predicting diabetic retinopathy. Scientific Reports 202111 7244. (https://doi. org/10.1038/s41598-021-83165-5)

22 Li JS, Wang T, Zuo JJ, Guo CN, Peng F, Zhao SZ, Li HH, Hou XQ, Lan Y, Wei YP, et al. Association of n-6 PUFAs with the risk of diabetic retinopathy in diabetic patients. Endocrine Connections 20209 1191-1201. (https://doi.org/10.1530/EC-20-0370)

23 Zuo J, Lan Y, Hu H, Hou X, Li J, Wang T, Zhang H, Zhang N, Guo C, Peng F, et al. Metabolomics-based multidimensional network biomarkers for diabetic retinopathy identification in patients with type 2 diabetes mellitus. BMJ Open Diabetes Research and Care 20219 e001443. (https://doi.org/10.1136/bmjdrc-2020-001443)

24 Wilkinson CP, Ferris FL, Klein RE, Lee PP, Agardh CD, Davis M, Dills D, Kampik A, Pararajasegaram R, Verdaguer JT, et al. Proposed international clinical diabetic retinopathy and diabetic macular edema disease severity scales. Ophthalmology 2003110 1677-1682. (https://doi.org/10.1016/S0161-6420(03)00475-5)

25 Eid S, Sas KM, Abcouwer SF, Feldman EL, Gardner TW, Pennathur S \& Fort PE. New insights into the mechanisms of diabetic complications role of lipids and lipid metabolism. Diabetologia 201962 1539-1549. (https://doi.org/10.1007/s00125-019-4959-1)

26 Li C, Sun YD, Yu GY, Cui JR, Lou Z, Zhang H, Huang Y, Bai CG, Deng LL, Liu P, et al. Integrated omics of metastatic colorectal cancer. Cancer Cell 202038 734.e9-747.e9. (https://doi.org/10.1016/j.ccell.2020.08.002)

27 Guo S \& Fraser MW. Propensity Score Analysis: Statistical Methods and Applications, 2nd ed. Thousand Oaks, CA, USA: SAGE Publications, Inc.

28 Svolba G. Data Preparation for Analytics Using SAS. Cary, NC: SAS Institute Inc.

29 Mao G, Nachman RM, Sun Q, Zhang X, Koehler K, Chen Z, Hong X, Wang G, Caruso D, Zong G, et al. Individual and joint effects of early-life ambient exposure and maternal prepregnancy obesity on childhood overweight or obesity. Environmental Health Perspectives 2017125 067005. (https://doi.org/10.1289/EHP261)

30 Liang W, Liang H, Ou L, Chen B, Chen A, Li C, Li Y, Guan W, Sang L, $\mathrm{Lu}$ J, et al. Development and validation of a clinical risk score to predict the occurrence of critical illness in hospitalized patients with COVID-19. JAMA Internal Medicine 2020180 1081-1089. (https://doi. org/10.1001/jamainternmed.2020.2033)

31 Zhang YB, Chen C, Pan XF, Guo J, Li Y, Franco OH, Liu G \& Pan A. Associations of healthy lifestyle and socioeconomic status with mortality and incident cardiovascular disease: two prospective cohort studies. BMJ 2021373 n604. (https://doi.org/10.1136/bmj.n604)

32 Xuan Q, Ouyang Y, Wang Y, Wu L, Li H, Luo Y, Zhao X, Feng D, Qin W, Hu C, et al. Multiplatform metabolomics reveals novel serum metabolite biomarkers in diabetic retinopathy subjects. Advanced Science 20207 2001714. (https://doi.org/10.1002/advs.202001714)

33 Arai C, Miyake M, Matsumoto Y, Mizote A, Yoshizane C, Hanaya Y, Koide K, Yamada M, Hanaya T, Arai S, et al. Trehalose prevents adipocyte hypertrophy and mitigates insulin resistance in mice with established obesity. Journal of Nutritional Science and Vitaminology 2013 59 393-401. (https://doi.org/10.3177/jnsv.59.393)

34 Yu B, Zheng Y, Alexander D, Morrison AC, Coresh J \& Boerwinkle E. Genetic determinants influencing human serum metabolome among African Americans. PLoS Genetics 201410 e1004212. (https://doi. org/10.1371/journal.pgen.1004212) https://ec.bioscientifica.com https://doi.org/10.1530/EC-21-0474 (c) 2022 The authors Published by Bioscientifica Ltd

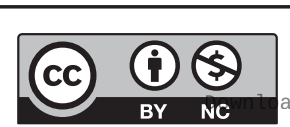

This work is licensed under a Creative Commons Attribution-NonCommercial 4.0 International License. ded from Bioscientifica, com at 04/26/2023 02:19:44PM 
35 Sanna S, van Zuydam N, Mahajan A, Kurilshikov A, Vila VA, Võsa U, Mujagic Z, Masclee A, Jonkers D, Oosting M, et al. Causal relationships among the gut microbiome, short-chain fatty acids and metabolic diseases. Nature Genetics 201951 600-605. (https://doi.org/10.1038/ s41588-019-0350-x)

36 Seo Y, Kingsley S, Walker G, Mondoux MA \& Tissenbaum HA. Metabolic shift from glycogen to trehalose promotes lifespan and healthspan in Caenorhabditis elegans. PNAS 2018115 E2791-E2800. (https://doi.org/10.1073/pnas.1714178115)

37 Zhang Y, Higgins CB, Fortune HM, Chen P, Stothard AI, Mayer AL, Swarts BM \& DeBosch BJ. Hepatic arginase 2 (Arg2) is sufficient to convey the therapeutic metabolic effects of fasting. Nature Communications 201910 1587. (https://doi.org/10.1038/s41467-01909642-8)

38 Yoshizane C, Mizote A, Arai C, Arai N, Ogawa R, Endo S, Mitsuzumi H $\&$ Ushio S. Daily consumption of one teaspoon of trehalose can help maintain glucose homeostasis: a double-blind, randomized controlled trial conducted in healthy volunteers. Nutrition Journal 20201968. (https://doi.org/10.1186/s12937-020-00586-0)

39 Lotfi P, Tse DY, Di Ronza A, Seymour ML, Martano G, Cooper JD, Pereira FA, Passafaro M, Wu SM \& Sardiello M. Trehalose reduces retinal degeneration, neuroinflammation and storage burden caused by a lysosomal hydrolase deficiency. Autophagy 201814 1419-1434. (https://doi.org/10.1080/15548627.2018.1474313)

40 Laskowska E \& Kuczyńska-Wiśnik D. New insight into the mechanisms protecting bacteria during desiccation. Current Genetics 202066 313-318. (https://doi.org/10.1007/s00294-01901036-z)
41 Moruno Algara M, Kuczyńska-Wiśnik D, Dębski J, StojowskaSwędrzyńska K, Sominka H, Bukrejewska M \& Laskowska E. Trehalose protects Escherichia coli against carbon stress manifested by protein acetylation and aggregation. Molecular Microbiology 2019112 866-880. (https://doi.org/10.1111/mmi.14322)

$42 \mathrm{Xu} \mathrm{J}$, Chen LJ, Yu J, Wang HJ, Zhang F, Liu Q \& Wu J. Involvement of advanced glycation end products in the pathogenesis of diabetic retinopathy. Cellular Physiology and Biochemistry 201848 705-717. (https://doi.org/10.1159/000491897)

43 Zong H, Ward M \& Stitt AW. AGEs, RAGE, and diabetic retinopathy. Current Diabetes Reports 201111 244-252. (https://doi.org/10.1007/ s11892-011-0198-7)

44 Manigrasso MB, Juranek J, Ramasamy R \& Schmidt AM. Unlocking the biology of RAGE in diabetic microvascular complications. Trends in Endocrinology and Metabolism 201425 15-22. (https://doi. org/10.1016/j.tem.2013.08.002)

45 Lieth E, Barber AJ, Xu B, Dice C, Ratz MJ, Tanase D \& Strother JM. Glial reactivity and impaired glutamate metabolism in short-term experimental diabetic retinopathy. Penn State Retina Research Group. Diabetes 199847 815-820. (https://doi.org/10.2337/diabetes.47.5.815)

46 Ola MS, Alhomida AS \& LaNoue KF. Gabapentin attenuates oxidative stress and apoptosis in the diabetic rat retina. Neurotoxicity Research 201936 81-90. (https://doi.org/10.1007/s12640-019-00018-w)

47 Moran EP, Wang Z, Chen J, Sapieha P, Smith LE \& Ma JX. Neurovascular cross talk in diabetic retinopathy: pathophysiological roles and therapeutic implications. American Journal of Physiology: Heart and Circulatory Physiology 2016311 H738-H749. (https://doi.org/10.1152/ ajpheart.00005.2016)

Received in final form 4 January 2022

Accepted 14 January 2022

Accepted Manuscript published online 14 January 2022
This work is licensed under a Creative Commons Attribution-NonCommercial 4.0 International License. ded from Bioscientifica.com at 04/26/2023 02:19:44PM 